\title{
SHARP TWO PARAMETER BOUNDS FOR THE LOGARITHMIC MEAN AND THE ARITHMETIC-GEOMETRIC MEAN OF GAUSS
}

\author{
Yu-Ming Chu, Miao-Kun Wang, Ye-Fang Qiu and XiaO-Yan Ma
}

Abstract. For fixed $s \geqslant 1$ and $t_{1}, t_{2} \in(0,1 / 2)$ we prove that the inequalities $G^{s}\left(t_{1} a+(1-\right.$ $\left.\left.t_{1}\right) b, t_{1} b+\left(1-t_{1}\right) a\right) A^{1-s}(a, b)>A G(a, b)$ and $G^{s}\left(t_{2} a+\left(1-t_{2}\right) b, t_{2} b+\left(1-t_{2}\right) a\right) A^{1-s}(a, b)>$ $L(a, b)$ hold for all $a, b>0$ with $a \neq b$ if and only if $t_{1} \geqslant 1 / 2-\sqrt{2 s} /(4 s)$ and $t_{2} \geqslant 1 / 2-$ $\sqrt{6 s} /(6 s)$. Here $G(a, b), L(a, b), A(a, b)$ and $A G(a, b)$ are the geometric, logarithmic, arithmetic and arithmetic-geometric means of $a$ and $b$, respectively.

Mathematics subject classification (2010): 26E20.

Keywords and phrases: Geometric mean, logarithmic mean, arithmetic-geometric mean of Gauss, arithmetic mean.

\section{REFERENCES}

[1] G. D. Anderson, M. K. Vamanamurthy and M. Vuorinen, Conformal Invariants, Inequalities, and Quasiconformal Maps, John Wiley \& Sons, New York, 1997.

[2] F. BowmAn, Introduction to Elliptic Functions with Application, Dover Publications, New York, 1961.

[3] P. BRACKen, An arithmetic-geometric mean inequality, Expo. Math. 19 (2001), 273-279.

[4] P. F. BYRD AND M. D. FRIEDMAN, Handbook of Elliptic Integrals for Engineers and Scientists, Springer-Verlag, New York, 1971.

[5] B. C. CARlson, Algorithms involving arithmetic and geometric means, Amer. Math. Monthly 78 (1971), 496-505.

[6] B. C. Carlson, The logarithmic mean, Amer. Math. Monthly 79 (1972), 615-618.

[7] B. C. CARlson And M. VuORInen, Inequalities of the AGM and the logarithmic mean, SIAM Review 33 (1991), 655-655.

[8] Y.-M. Chu AND M.-K. WANG, Optimal inequalities between harmonic, geometric, logarithmic, and arithmetic-geometric means, J. Appl. Math. 2011, Article ID 618929, 9 pages.

[9] Y.-M. CHU, M.-K. WANG AND Z.-K. WANG, Best possible inequalities among harmonic, geometric, logarithmic and Seiffert means, Math. Inequal. Appl. 15 (2012), 415-422.

[10] W. FECHNER, On some functional inequalities related to the logarithmic mean, Acta Math. Hungar 128 (2010), 36-45.

[11] H. Kos AKI, Arithmetic-geometric mean and related inequalities for operators, J. Funct. Anal. 156 (1998), 429-451.

[12] T. P. LIN, The power mean and the logarithmic mean, Amer. Math. Monthly 81 (1974), 879-883.

[13] L. G. LuCHT, On the arithmetic-geometric mean inequality, Amer. Math. Monthly 102 (1995), 739740 .

[14] E. Neuman, The weighted logarithmic mean, J. Math. Anal. Appl. 188 (1994), 885-900.

[15] E. Neuman And J. SÁndor, On the Schwab-Borchardt mean, Math. Pannon. 14 (2003), $253-266$.

[16] J. SÁndor, On the identric and logarithmic means, Aequationes Math. 40 (1990), 261-270.

[17] J. SÁndor, On certain inequalities for means, J. Math. Anal. Appl. 189 (1995), 602-606.

[18] J. SÁndor, On certain inequalities for means II, J. Math. Anal. Appl. 199 (1996), 629-635.

[19] M. K. Vamanamurthy and M. Vuorinen, Inequalities for means, J. Math. Anal. Appl. 183 (1994), 155-166. 